\title{
Oxidase activity of plasma ceruloplasmin in obstructive sleep apnea patients
}

\author{
MACIEJ CYMERYS ${ }^{1}$, SZCZEPAN COFTA2, EWA BEATA WYSOCKA ${ }^{3}$, MARCIN NOWICKI $^{3}$, \\ JOANNA SULIBURSKA ${ }^{4}$, WIESEAW BRYL ${ }^{1}$, LECH TORLINSSKI \\ 'Department of Internal Medicine, Metabolic Disorders and Hypertension, Poznan University of Medical Sciences, Poznan, Poland \\ ${ }^{2}$ Department of Pulmonology, Allergology and Respiratory Oncology, Poznan University of Medical Sciences, Poznan, Poland \\ ${ }^{3}$ Chair of Chemistry and Clinical Biochemistry, Department of Clinical Biochemistry and Laboratory Medicine, Poznan University \\ of Medical Sciences, Poznan, Poland \\ ${ }^{4}$ Department of Human Nutrition and Hygiene, Poznan University of Life Sciences, Poznan, Poland
}

\begin{abstract}
Aim of the study: To analyze the oxidase activity of ceruloplasmin (CP) along with copper concentrations, in the blood of different stage obstructive sleep apnea (OSA) patients.

Material and methods: Sixty normoglycemic males aged 35-65 without any acute or chronic disease were included into the study. EMBLA 54000 system was used to establish the apnea/hypopnea index (AHI) and to divide patients into groups: OSA1 with AHI 5-15 $(n=20)$; OSA2 with AHI 16-30 $(n=20)$; OSA3 with AHI $\geq 31(n=20)$. The control group $(n=22)$ had no OSA suspicion. Fasting plasma lipid profile, serum insulin, plasma ceruloplasmin concentration (Cp-c), serum copper $(C u)$ concentrations and oxidase activity of plasma ceruloplasmin (Cp-oa) by Schosinsky's method were estimated.

Results: 1. All three OSA groups did not differ in systolic (SBP) and diastolic (DBP) blood pressure and $C p$-c. Increased Cp-oa was observed in OSA-1 and OSA-2 groups, although the increased Cu was observed in OSA-1, then decreased Cu was found in OSA-2 and OSA-3.

2. OSA-1 patients were found to show positive relationships between $C p$-oa and $S B P, D B P$ and $C u$, of which SBP correlated independently from others. In OSA-2 group, Cp-oa was rather affected by AHI. OSA-3 subjects presented positive correlations of SBP\&BMI and SBP\&AHI with an independent influence of $A H I$.

Conclusions: We suggest some mobilization of plasma CP activity in early stages of OSA. The oxidase activity of plasma ceruloplasmin in normoglycemic OSA patients may reflect some compensation for an increased activity of the sympathetic nervous system (elevated blood pressure) until there is severe OSA pathology.
\end{abstract}

Key words: ceruloplasmin, copper, obstructive sleep apnea, blood pressure, oxidase activity.

(Centr Eur J Immunol 2013; 38 (4): 511-517)

\section{Introduction}

Obstructive sleep apnea (OSA) affects $2-4 \%$ of the adult world population $[1,2]$ and is characterised by repetitive episodes of complete or partial upper airway obstruction during sleep resulting in decreasing oxygenation of blood and arousals. Repeated hypoxemia along with awakenings are discussed to increase, among others, an activity of the sympathetic nervous system, oxidative stress and endothelial dysfunction, thus a cardiovascular aspect of OSA is under intensive investigation [3].

On the one hand, we realize the coexistence of cardiovascular risk factors and OSA in excessive body mass patients, and on the other hand, recurrent apnea/hypopnea episodes and cyclical alterations of arterial oxygen saturation might be involved in the development of hypertension, diabetes and lipid disturbances in obstructive sleep apnea patients [4].
Researchers are interested in antioxidant defense and its contributions in OSA. In the advanced obstructive sleep apnea syndrome, an oxidative stress might be derived directly from OSA pathology and be related to cardiovascular risk factors or cardiovascular disease developed [5].

The elementary model of oxygen free radical production is an incomplete reduction of oxygen to water during electron transport chain in mitochondria, with a possible releasing of superoxide radicals $\mathrm{O}_{2}^{-}$out of the organelle [6]. Copper and zinc-containing superoxide dismutase, SOD-1, located in the cytoplasm, catalyzes the dismutation of two superoxide radicals $\mathrm{O}_{2}^{-}$due to the following reaction: $\mathrm{O}_{2}^{-}+$ $\mathrm{O}_{2}^{-}+2 \mathrm{H}^{+} \rightarrow \mathrm{H}_{2} \mathrm{O}_{2}+\mathrm{O}_{2}$, in which less danger hydrogen peroxide is formed [6]. When limiting availability of $\cdot \mathrm{O}_{2}^{-}$, it is possible to prevent a generation of hydroxyl radical $\cdot \mathrm{OH}$ in the reaction catalyzed by transient metal ions (Fenton and Haber-Weiss reactions). While erythrocyte SOD-1 activity

Correspondence: Maciej Cymerys, MD, PhD, Department of Internal Medicine, Metabolic Disorders and Hypertension, Poznan University of Medical Sciences, Szamarzewskiego 84, 60-569 Poznan, Poland, e-mail: maciejcymerys@wp.pl 
is considered to represent essential intracellular enzymatic antioxidant defense, ceruloplasmin expresses lower SODlike activity extracellular in blood [7].

Ceruloplasmin ( $\mathrm{Cp}$ ) belongs to the $\alpha_{2}$-globulin fraction of human serum, produced in the liver as an apoprotein, and after binding 6-7 copper ions $\mathrm{Cp}$ is secreted to the blood. This protein, collecting $70-90 \%$ of total $\mathrm{Cu}$ in plasma, is a major plasma oxidase which complexes copper ions, what may limit the availability of $\mathrm{Cu}$ for Fenton reaction [7] and is capable of oxidizing the biogenic amines, including adrenalin and noradrenalin, limiting their biological function [8]. Ceruloplasmin regulates copper and iron metabolism, as with its ferroxidase activity can catalyze the oxidation from $\mathrm{Fe}^{2+}$ to $\mathrm{Fe}^{3+}$. The final result is the decrease in the availability of ions ${ }^{2+}$ for Fenton reaction, thus the limited generation of hydroxyl radical $\cdot \mathrm{OH}$ extracellularly [9].

An evident inflammatory process is generally induced by severe harmful factors, i.e. microorganism and tissue injury, and is characterized by elevated acute phase proteins, including ceruloplasmin. Inflammatory markers express biochemical response during immunological adaptive mechanisms, thus we discuss a number of biogenic amines and different acute phase proteins. Mediators of the inflammatory process also trigger neuro-humoral response with an increasing catecholamine level in blood. Each inflammation is accompanied by oxidative stress [10].

Mild inflammatory reaction has been recognized in several disorders, including obesity, atherosclerosis, type 2 diabetes and metabolic syndrome, and inflammatory marker high sensitivity C-reactive protein (hsCRP) has been proposed among predictive factors for cardiovascular disease. From an immunological point of view, low-grade inflammation may reflect a predominance of processes to limit inflammatory reaction on cellular damage [11].

Ceruloplasmin with its unique multiple activity may play an important role in systemic response to obstructive sleep apnea. Enzyme activity may be an individual feature, but contributed by necessary co-factors. The plasma level of the trace element along with the main copper-containing plasma protein, ceruloplasmin $(\mathrm{Cp})$ with its possible preventive function, could complete our knowledge about pathobiochemistry of OSA.

The aim of the study was to analyze the oxidase activity of ceruloplasmin ( $\mathrm{CP}-\mathrm{a})$ along with copper and ceruloplasmin concentrations, in the blood of different stageOSA patients.

\section{Material and methods}

\section{Subjects and settings}

The study was performed in accordance with the Declaration of Helsinki for Human Research and the study protocol was accepted by the Bioethical Committee of the University of Medical Sciences in Poznan, Poland. Every subject gave informed consent to the study procedure.

Non-smoking Caucasians suspected of OSA, without any acute or chronic disease, using neither special diet nor medication, were subjected to clinical, biochemical and full-night polysomnographic examinations. Eighty-two males, residents of Wielkopolska province, aged 35-65, with body mass index (BMI) $25.0-40.0 \mathrm{~kg} / \mathrm{m}^{2}$, participated in the study. Moreover, the inclusion criteria were: normal glucose tolerance confirmed during the oral glucose tolerance test $[12,13]$, complete blood count with no symptoms of anemia or inflammation and plasma ceruloplasmin concentrations within in the range 0.150 to $0.400 \mathrm{~g} / 1$ [14]. The apnea/hypopnea index (AHI) was to divide patients into groups: OSA1 with AHI 5-15 $(n=20)$; OSA2 with AHI $16-30(n=20)$; OSA3 with AHI $\geq 31(n=20)$. The control group, $\mathrm{C}(n=22)$, was composed of age, BMI and glycemic status-similar individuals without OSA.

\section{Measurements}

Subjects underwent complete physical examination, including the calculation of body mass index (BMI, $\mathrm{kg} / \mathrm{m}^{2}$ ) and the measurement of systolic (SBP) and diastolic (DBP) arterial blood pressure. The arterial blood pressure was assessed twice on the left arm with an appropriate cuff size, using sphygmomanometer MEDEL Palm Pro 91431 (Medel, Italy), after at least 15 minutes' rest in a sitting position, and the result was expressed as the mean value of two measurements.

The full-night polysomnography (Remlogic, EMBLA S4000 system, Denver, CO, USA) was performed in the Sleep Laboratory of the Department of Pulmonology, Allergology and Respiratory Oncology, according to the standard diagnostic procedure.

Ulnar venous blood was drawn after overnight fast for all parameters listed below, and then the oral glucose tolerance test (OGTT) was performed to find normal glucose tolerance subjects. Prediabetic (impaired fasting glycemia and impaired glucose tolerance) and diabetic males were excluded from the study. Normal glucose tolerance was defined by fasting glucose (G-0') $<5.60 \mathrm{mmol} / \mathrm{l}$ along with 2-h glycemia (G-120') < 7.80 mmol/l, during 75-g OGTT.

The concentrations of plasma glucose and lipid profile: total cholesterol (T-C), triglycerides (TG) and high-density lipoprotein cholesterol (HDL-C) were measured by enzymatic methods (Dimension Xpand Plus Systems, Siemens Healthcare Diagnostics, Deerfield, IL, USA). Low-density lipoprotein cholesterol (LDL-C) was calculated using Friedewald's formula: LDL-C $=[$ T-C $]-[$ HDL-C $]-$ [TG/2.2].

The concentration of insulin was measured by ELISA method (BioSource, Nivelles, Belgium) using the Sunrise microplater reader (Tecan Group, Männedorf, Switzerland), with sensitivity of $0.15 \mathrm{mU} / \mathrm{l}$. The intra- and 
inter-assay coefficients of variation (CV) were $3.8 \%$ and $5.4 \%$, respectively. Homeostatic model assessment for insulin resistance was calculated as HOMA-IR $=\mathrm{G} 0$ ' $\times$ Ins/22.5.

The plasma ceruloplasmin concentration was determined nephelometrically using Dade Behring Nephelometer II (Siemens Healthcare Diagnostics, Deerfield, IL, USA).

Serum copper $(\mathrm{Cu})$ concentrations were measured in duplicate by flame atomic absorption spectrometry Zeiss AAS-3 (Carl Zeiss, Jena, Germany).

The reference sera level 1 and level 2 (Randox Laboratories, Crumlin, Antrim, UK) were used for monitoring the accuracy of the above determinations.

The oxidase activity of plasma ceruloplasmin EC.1. 16.3.1. (Cp-oa) was evaluated spectrophotometrically after the incubation with o-dianisidine dihydrochloride as a substrate, according to Schosinsky et al. [15] using Sigma reagents (Sigma-Aldrich Co, St. Louis, MO, USA) and Specord M40 (Carl Zeiss, Jena, Germany) with 3.5\% of intra-assay and $4.7 \%$ of inter-assay CV calculated.

\section{Statistical analysis}

Statistical calculation was performed using Statistica 10.0 for Windows program. The distributions of clinical and biochemical parameters were verified by Shapiro-Wilk's test. All results are presented as a median along with lower and upper quartiles given in parentheses. Differences among Controls, OSA-1, OSA-2 and OSA-3 groups were evaluated using nonparametric Kruskal-Wallis ANOVA \& median test, and then multiple comparison was performed using post hoc Dunn test. Spearman's correlation coefficient $(\mathrm{R})$ described relationships. Multiple regression analysis was performed in selected groups and the results were expressed as $\beta, \mathrm{R}$ and $\mathrm{R}^{2}$ coefficients. Statistical significance was established when $p<0.05$.

\section{Results}

The clinical and laboratory characteristics of the study groups is presented in Table 1.

Subjects did not differ in their age, BMI, glucose levels, insulin concentration and HOMA-IR, as well as con-

Table 1. The characteristics of the study groups. Data are presented as a median and an interquartile range (in parenthesis). The differences among the groups verified by Kruskal-Wallis test (details in the text) are marked with an asterisk*

\begin{tabular}{|c|c|c|c|c|c|c|}
\hline & $\begin{array}{l}\text { Controls } \\
(n=22)\end{array}$ & $\begin{array}{c}\text { OSA-1 } \\
(n=20))\end{array}$ & $\begin{array}{c}\text { OSA-2 } \\
(n=20)\end{array}$ & $\begin{array}{c}\text { OSA-3 } \\
(n=20)\end{array}$ & $\begin{array}{c}\text { Differences } \\
\text { among All } \\
(n=82)\end{array}$ & $\begin{array}{c}\text { Differences } \\
\text { among OSA } \\
(n=60)\end{array}$ \\
\hline Age [years] & $50.0(43.0-56.5)$ & $49.5(43.5-57.5)$ & $50.0(45.0-56.5)$ & $51.0(44.0-55.5)$ & & \\
\hline AHI [events/h] & $1.70(0.90-2.80)$ & $8.90(6.40-12.3)$ & $22.1(19.2-25.8)$ & $47.0(38.8-73.2)$ & $*$ & $*$ \\
\hline BMI $\left[\mathrm{kg} / \mathrm{m}^{2}\right]$ & $31.5(28.8-34.3)$ & $32.0(28.0-34.7)$ & $31.6(28.6-34.0)$ & $31.7(29.7-33.9)$ & & \\
\hline $\mathrm{SBP}[\mathrm{mm} \mathrm{Hg}]$ & $130(123-140)$ & $140(120-160)$ & $140(130-140)$ & $130(130-140)$ & $*$ & \\
\hline DBP [mm Hg] & $80(80-90)$ & $81(80-97)$ & $90(80-90)$ & $90(80-90)$ & $*$ & \\
\hline G-0' $[\mathrm{mmol} / \mathrm{l}]$ & $5.12(4.90-5.26)$ & $5.06(4.87-5.28)$ & $5.11(4.89-5.35)$ & $5.23(5.02-5.36)$ & & \\
\hline G-120' $[\mathrm{mmol} / \mathrm{l}]$ & $5.91(5.34-6.58)$ & $5.78(5.50-6.56)$ & $5.84(5.48-6.86)$ & $5.88(5.17-6.56)$ & & \\
\hline Ins-0' [mU/l] & $18.1(12.9-22.3)$ & $16.1(11.4-25.5)$ & $16.8(11.1-22.1)$ & $19.5(13.3-22.3)$ & & \\
\hline HOMA-IR & $3.97(3.02-5.01)$ & $3.51(2.61-5.82)$ & $3.72(2.53-5.20)$ & $4.24(2.96-5.18)$ & & \\
\hline T-C [mmol/l $]$ & $5.33(4.65-6.44)$ & $5.50(4.95-6.19)$ & $5.48(4.83-5.75)$ & $5.49(4.73-6.23)$ & & \\
\hline $\mathrm{TG}[\mathrm{mmol} / \mathrm{l}]$ & $1.14(0.81-2.16)$ & $1.46(1.02-2.06)$ & $1.19(0.79-2.38)$ & $1.59(1.21-2.22)$ & & \\
\hline HDL-C [mmol/l] & $1.26(1.15-1.54)$ & $1.09(1.06-1.36)$ & $1.07(1.00-1.37)$ & $1.05(0.97-1.17)$ & $*$ & \\
\hline LDL-C [mmol/l] & $3.52(2.67-4.23)$ & $3.51(3.22-4.49$ & $3.28(2.94-3.99)$ & $3.61(2.73-4.16)$ & & \\
\hline Cp-c [g/l] & $0.240(0.220-0.258)$ & $0.273(0.243-0.287)$ & $0.265(0.241-0.302)$ & $0.268(0.255-0.285)$ & $*$ & \\
\hline $\mathrm{Cu}[\mu \mathrm{mol} / \mathrm{l}]$ & $15.21(13.93-16.70)$ & $16.77(14.87-18.23)$ & $13.23(11.79-14.76)$ & 11.95 (11.16-14.19) & $*$ & $*$ \\
\hline Cp-oa [U/I] & $89.55(76.90-105.0)$ & $101.0(81.50-128.8)$ & $126.95(95.1-156.0)$ & $103.24(83.00-116.9)$ & $*$ & \\
\hline
\end{tabular}




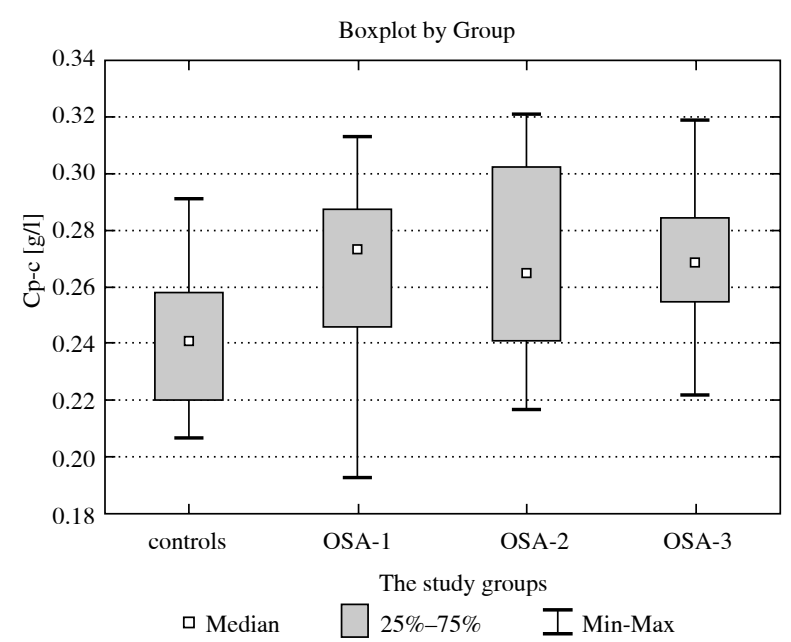

Fig. 1. The comparison of plasma ceruloplasmin concentration $(\mathrm{Cp}-\mathrm{c})$ in the study groups: the boxplots of the values are shown in the graph, and the results of analysis of variance by Kruskal-Wallis test and post hoc analysis using Dunn test, are presented in Table 2

centrations of total cholesterol, triglycerides and LDL-cholesterol. The decreasing HDL-C was observed according to OSA diagnosis (Kruskal-Wallis test $p=0.0187$ ) with the lowest levels in OSA-3 group as compared to Controls (Dunn test $p=0.0116)$. The subjects differed as to blood pressure: SBP (Kruskal-Wallis test $p=0.0194$ ) and DBP (Kruskal-Wallis test $p=0.0375$ ). Increased SBP was found in OSA-2 group as compared to Controls (Dunn test $p=0.0454$ ). There were no differences in post hoc analysis concerning DBP.

Despite settings of the study and inclusion criterion of plasma ceruloplasmin concentration within reference values, OSA-2 and OSA-3 groups presented increased Cp-c as compared to Control group (Fig. 1, Table 2).

The serum copper concentrations in Controls and OSA-1 group remained within references for females 13-24 $\mu \mathrm{mol} / 1$ and males 11-22 $\mu \mathrm{mol} / 1$ [14]. The highest serum copper concentrations, however, not different from Controls, were found in OSA-1 group. OSA-2 and OSA-

Table 2. The comparison of plasma ceruloplasmin concentration (Cp-c) in the study groups: the results of analysis of variance by Kruskal-Wallis test and post hoc analysis using Dunn test

\begin{tabular}{lcccc}
\hline & Controls & OSA-1 & OSA-2 & OSA-3 \\
\hline Controls & & 0.0551 & $\mathbf{0 . 0 4 3 3}$ & $\mathbf{0 . 0 1 8 1}$ \\
\hline OSA-1 & 0.0551 & & 1.0000 & 1.0000 \\
\hline OSA-2 & $\mathbf{0 . 0 4 3 3}$ & 1.0000 & & 1.0000 \\
\hline OSA-3 & $\mathbf{0 . 0 1 8 1}$ & 1.0000 & 1.0000 & \\
\hline
\end{tabular}

Cp-c: Kruskal-Wallis test: $p=0.0082$

Dunn test: Multiple Comparisons p values (2-tailed):

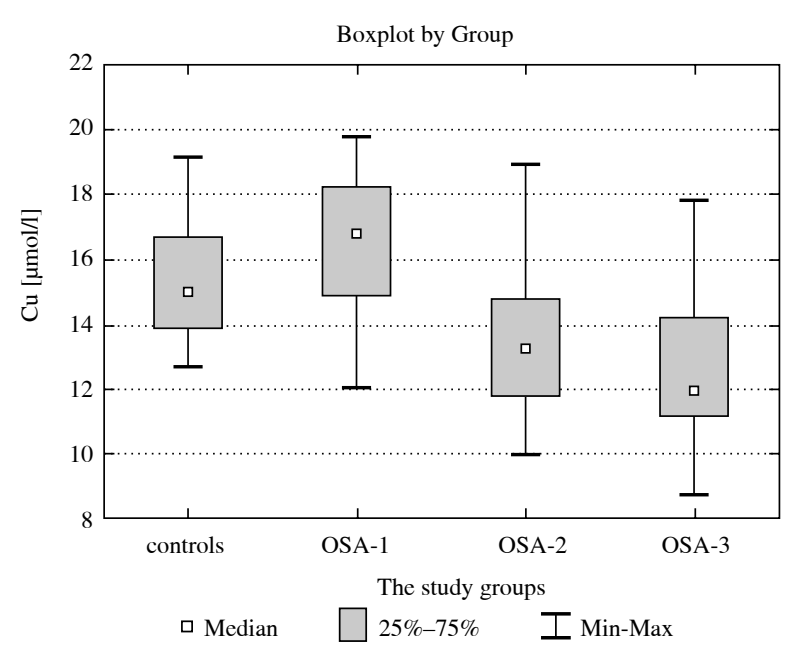

Fig. 2. The comparison of serum copper concentration $\mathrm{Cu}$ in the study groups: the boxplots of the values are shown in the graph, and the results of analysis of variance by Kruskal-Wallis test and post hoc analysis using Dunn test, are presented in Table 3

3 subjects presented decreasing values comparing with both Controls and OSA-1 men (Fig. 2), and some individuals were found below references mentioned above (Table 3).

The different oxidase activities of plasma ceruloplasmin were found in the groups studied. The general tendency for increased Cp-oa in obstructive sleep apnea was observed and OSA-2 subjects presented the highest values, while OSA-1 and OSA-3 groups showed the similar activities (Fig. 3, Table 4).

Statistically important correlations are presented in Table 5.

The multiple regression analysis showed SBP to be an independent factor affecting oxidase activity of plasma ceruloplasmin $\left(\beta=0.798 ; \mathrm{R}=0.814 ; \mathrm{R}^{2}=0.663 ; p=0.0005\right)$ in OSA-1 group. Evaluating influences on systolic blood pressure, AHI was indicated to correlate independently with $\operatorname{SBP}\left(\beta=0.627 ; \mathrm{R}=0.778 ; \mathrm{R}^{2}=0.605 ; p=0.0004\right)$ in OSA-3 group.

Table 3. The comparison of serum copper concentration $\mathrm{CCu}$ in the study groups: the results of analysis of variance by Kruskal-Wallis test and post hoc analysis using Dunn test

\begin{tabular}{lcccc}
\hline & Controls & OSA-1 & OSA-2 & OSA-3 \\
\hline Controls & & 1.0000 & 0.0842 & $\mathbf{0 . 0 0 3 2}$ \\
\hline OSA-1 & 1.0000 & & 0.0014 & $<0.0001$ \\
\hline OSA-2 & 0.0842 & 0.0014 & & 1.0000 \\
\hline OSA-3 & $\mathbf{0 . 0 0 3 2}$ & $<\mathbf{0 . 0 0 0 1}$ & 1.0000 & \\
\hline
\end{tabular}

Cu: Kruskal-Wallis test: $p=0.0000$

Dunn test: Multiple Comparisons p values (2-tailed): 


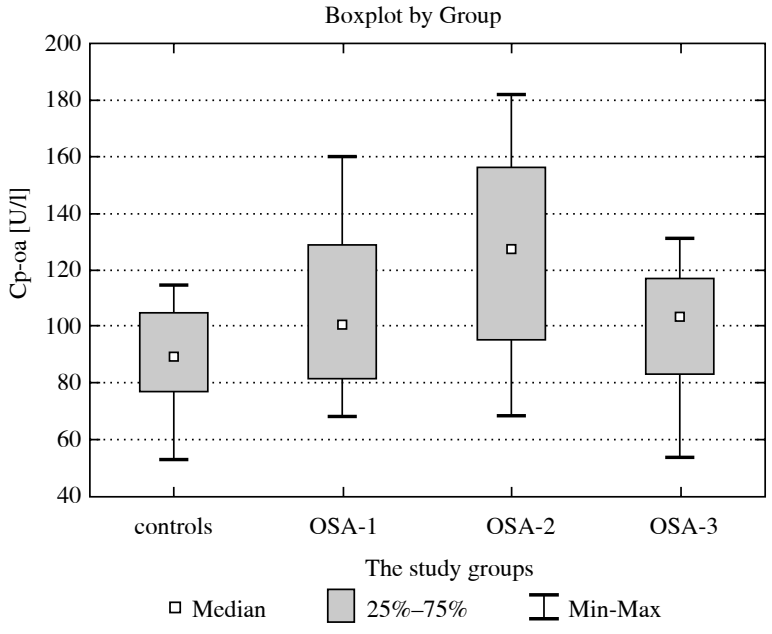

Fig. 3. The comparison of oxidase activity of plasma ceruloplasmin (Cp-oa) in the study groups: the boxplots of the values are shown in the graph, and the results of analysis of variance by Kruskal-Wallis test and post hoc analysis using Dunn test, are presented in Table 4

\section{Discussion}

Experimental and clinical studies have reported an association between sleep deprivation and increased circulating levels of inflammatory mediators (i.e. interleukins, acute phase proteins and prostaglandins) $[16,17]$. These alterations may contribute to the development of metabolic
Table 4. The comparison of oxidase activity of plasma ceruloplasmin (Cp-oa) in the study groups: the results of analysis of variance by Kruskal-Wallis test and post hoc analysis using Dunn test

\begin{tabular}{lcccc}
\hline & Controls & OSA-1 & OSA-2 & OSA-3 \\
\hline Controls & & 0.4398 & $\mathbf{0 . 0 0 2 5}$ & 0.8037 \\
\hline OSA-1 & 0.4398 & & 0.5427 & 1.0000 \\
\hline OSA-2 & $\mathbf{0 . 0 0 2 5}$ & 0.5427 & & 0.2872 \\
\hline OSA-3 & 0.8037 & 1.0000 & 0.2872 & \\
\hline
\end{tabular}

Cp-oa: Kruskal-Wallis test: $p=0.0059$

Dunn test: Multiple Comparisons p values (2-tailed):

and endothelial dysfunction, atherosclerosis and cardiovascular disorders associated with OSA [18]. However, conflicting reports of the concentrations of the most popular marker - C-reactive protein (CRP) in the blood of OSA patients, are available. Increased CRP due to the severity of the disease [19] and no relationships [20] are documented.

There are no data concerning ceruloplasmin concentration and its oxidase activity in OSA available. Our study discusses oxidase activity of $\mathrm{Cp}$ together with concentrations of ceruloplasmin and copper in blood for the first time, especially in different-stage OSA men.

In our study, increased $\mathrm{Cp}-\mathrm{c}$ was observed in moderate and severe OSA as compared to Controls, presenting non-inflammatory levels by the definition. In the population-based study exploring the effects of low-grade inflammation and hypertension on acute coronary events, we

Table 5. The correlations in the study groups. Spearman's coefficient $(\mathrm{R})$ is used to describe the relationship if it is statistically significant $(p<0.05)$; NS - no significance

\begin{tabular}{|c|c|c|c|c|c|c|}
\hline & $\begin{array}{l}\text { OSA-0 } \\
n=22\end{array}$ & $\begin{array}{l}\text { OSA-1 } \\
n=20\end{array}$ & $\begin{array}{l}\text { OSA-2 } \\
n=20\end{array}$ & $\begin{array}{l}\text { OSA-3 } \\
n=20\end{array}$ & $\begin{array}{c}\text { OSA-(1+2) } \\
n=40\end{array}$ & $\begin{array}{c}\text { OSA- }(1+2+3) \\
n=60\end{array}$ \\
\hline Cp-oa \& AHI & NS & NS & $\begin{array}{c}\mathrm{R}=0.63 \\
p=0.0027\end{array}$ & NS & $\begin{array}{c}\mathrm{R}=0.38 \\
p=0.0162\end{array}$ & NS \\
\hline Cp-oa \& SBP & NS & $\begin{array}{c}\mathrm{R}=0.83 \\
p<0.0001\end{array}$ & NS & NS & $\begin{array}{c}\mathrm{R}=0.54 \\
p=0.0003\end{array}$ & $\begin{array}{c}\mathrm{R}=0.43 \\
p=0.0005\end{array}$ \\
\hline Cp-oa \& DBP & NS & $\begin{array}{c}\mathrm{R}=0.57 \\
p=0.0083\end{array}$ & NS & NS & $\begin{array}{c}\mathrm{R}=0.48 \\
p=0.0018\end{array}$ & $\begin{array}{c}\mathrm{R}=0.37 \\
p=0.0039\end{array}$ \\
\hline Cp-oa \& Cu & $\begin{array}{c}\mathrm{R}=0.97 \\
p<0.0001\end{array}$ & $\begin{array}{c}\mathrm{R}=0.55 \\
p=0.0128\end{array}$ & $\begin{array}{c}\mathrm{R}=0.81 \\
p<0.0001\end{array}$ & $\begin{array}{c}\mathrm{R}=0.92 \\
p<0.0001\end{array}$ & $\begin{array}{c}\mathrm{R}=0.36 \\
p=0.0222\end{array}$ & $\begin{array}{c}\mathrm{R}=0.53 \\
p<0.0001\end{array}$ \\
\hline Cp-oa \& Cp-c & $\begin{array}{c}\mathrm{R}=0.52 ; \\
p=0.0124\end{array}$ & NS & NS & $\begin{array}{c}\mathrm{R}=0.69 ; \\
p=0.0007\end{array}$ & NS & NS \\
\hline SBP \& BMI & NS & NS & NS & $\begin{array}{c}\mathrm{R}=0.63 ; \\
p=0.0029\end{array}$ & NS & NS \\
\hline SBP \& AHI & NS & NS & NS & $\begin{array}{c}\mathrm{R}=0.66 \\
p=0.0014\end{array}$ & NS & NS \\
\hline Cu \& AHI & NS & NS & NS & NS & $\begin{array}{l}\mathrm{R}=-0.48 \\
p=0.0020\end{array}$ & $\begin{array}{l}\mathrm{R}=-0.49 \\
p<0.0001\end{array}$ \\
\hline Cu \& Cp-c & $\begin{array}{c}\mathrm{R}=0.48 \\
p=0.0235\end{array}$ & NS & NS & NS & NS & NS \\
\hline
\end{tabular}


can find increased plasma Cp concentrations when blood pressure was $\geq 160 / 100 \mathrm{~mm} \mathrm{Hg}$ comparing with normotensive and borderline patients, even if $\mathrm{Cp}$ was also presented within references [21].

Whole-body copper metabolism and indexes of copper status, including plasma $\mathrm{Cu}$, ceruloplasmin concentration and activity, and urinary copper excretion are not affected by nutritional factors, when dietary copper intake goes from 0.8 to $7.5 \mathrm{mg} /$ day. However, several conditions and disorders influence copper metabolism, such as pregnancy, inflammation, metabolic defects, including Menkes syndrome and Wilson disease, and total parenteral nutrition. A series of studies conducted on different populations (young and elderly, men and women) under a variety of conditions (i.e. pregnancy) with various diets, showed that a 10 -fold increase in dietary copper resulted in only doubling the absorption [22, 23]. Thus, the settings of this study were followed to meet comparable steady-state conditions for subjects. The between-groups changes of serum copper levels, concerning advanced OSA pathology, have been accepted to suggest some feature of severity of OSA.

There are few studies on serum copper in OSA patients documenting no changes [24] and increased $\mathrm{Cu}$ concentration in the severe stage of OSA but along with a consecutive hsCRP increase [25]. Other possible inflammatory-associated disorders, in which low total serum copper was suggested to predict high intracranial pressure (sensitivity $86 \%$ and specificity $73 \%$ ) after traumatic brain injury [26] could be mentioned.

Comparing a combination of parameters: $\mathrm{Cp}$-oa and $\mathrm{Cu}$, among groups, we can find that mild OSA-1 subjects presented elevated $\mathrm{Cu}$ concentrations along with a tendency to increase $\mathrm{Cp}$-oa comparing with Controls. More advanced, moderate, OSA-2 group was found to have increased Cp-oa together with a decreased copper level, while severe OSA-3 subjects presented decreased $\mathrm{Cu}$ and $\mathrm{Cp}$-oa back at the level of OSA-1. Because of the significant positive correlations of $\mathrm{Cp}$-oa \& $\mathrm{Cu}$ : almost perfect in Control and OSA-3 groups, strong in OSA-2 and good (but the lowest) in OSA-1, and Cp-oa positively related to SBP mainly in OSA-1 group, the most interesting solution could be a possible mobilization of plasma ceruloplasmin activity in mild-moderate OSA in the context of elevated blood pressure. As it was observed, despite a relatively decreased serum copper concentration in OSA-2 subjects, this may suggest some modification of ceruloplasmin far from the only copper ion content. Severe OSA pathology was presented with decreased $\mathrm{Cp}$-oa and $\mathrm{Cu}$ levels together, comparing to OSA-1 and OSA-2 patients.

Baysal et al. evaluated several plasma factors, mainly in the context of their antioxidant function, in mild-tosevere OSA patients together, and increased ferroxidase activity of $\mathrm{Cp}$ was observed in the obstructive sleep apnea group as compared to the control group. The two-fold higher standard deviation for $\mathrm{Cp}$ activity was calculated in OSA as compared to controls [27], what proves some variability of $\mathrm{Cp}$ activities and may confirm our results obtained in different stages of OSA.

The researchers were able to find a decreased or increased activity of $\mathrm{Cp}$ in some pathologies [28], however the nature and way of modification are not known. Studies on copper sites in Cp showed that the domains could be affected by $\mathrm{pH}$ and other ions, especially chloride [29]. The idea of immunologic contribution may accompany the typical chemical changes of copper sites in the molecule. The additional relationships between Cp-oa and SBP or DBP in obstructive sleep apnea patients could confirm metabolic disturbances in hypertensive patients, like an elevated concentration of resistin or TNF- $\alpha$ being considered a missing link. If it is possible that a mechanism of hypertension could develop/start immune cells actions and production of cytokines affecting, i.e. liver function resulting in increased production of C-reacting protein (see above), it is also possible that during the same elevated blood pressure pathology cytokines may result in the production of some factors affecting $\mathrm{Cp}$ activity. The study concerning oxidase activity and hypertension were performed only on elderly people, and elevated $\mathrm{Cp}$ activities had been decreased during hypotensive therapy using perindopril [30].

\section{Conclusions}

We suggest some mobilization of plasma ceruloplasmin activity in mild and moderate stages of OSA. The oxidase activity of plasma ceruloplasmin in normoglycemic OSA patients may reflect some compensation for elevated blood pressure (increased activity of sympathetic nervous system) until there is severe OSA pathology.

\section{The authors declare no conflict of interest.}

\section{References}

1. Young T, Palta M, Dempsey J, et al. (1993): The occurrence of sleep-disordered breathing among middle-aged adults. N Engl J Med 328: 1230-1235.

2. Young T, Palta M, Dempsey J, et al. (2009): Burden of sleep apnea: rationale, design, and major findings of the Wisconsin Sleep Cohort study. WMJ 108: 246-249.

3. Garvey JF, Taylor CT, McNicholas WT (2009): Cardiovascular disease in obstructive sleep apnoea syndrome: the role of intermittent hypoxia and inflammation. Eur Respir J 33: 1195-1205.

4. McNicholas WT, Bonsigore MR; Management Committee of EU COST ACTION B26 (2007): Sleep apnea as an independent risk factor for cardiovascular disease: Current evidence, basic mechanisms and research priorities. Eur Respir J 29: 156-178.

5. Suzuki YJ, Jain V, Park AM, Day RM (2006): Oxidative stress and oxidant signaling in obstructive sleep and associ- 
ated cardiovascular diseases. Free Radic Biol Med 40: 16831692.

6. Valko M, Leibfritz D, Moncol J, et al. (2007): Free radicals and antioxidants in normal physiological function and human disease. Int J Biochem Cell Biol 39: 44-84.

7. Arredondo M, González M, Olivares M, et al. (2008): Ceruloplasmin, an indicator of copper status. Biol Trace Elem Res 123: 261-269.

8. Gutteridge JM, Stocks J (1981): Ceruloplasmin: physiological and pathological perspectives. Crit Rev Clin Lab Sci 14: 257-329.

9. Giurgea N, Constantinescu MI, Stanciu R, et al. (2005): Ceruloplasmin - acute-phase reactant or endogenous antioxidant? The case of cardiovascular disease. Med Sci Monit 11: RA48-51.

10. Całkosiński I, Dobrzyński M, Całkosińska M, et al. (2009): Characterization of an inflammatory response. Postepy Hig Med Dosw 63: 395-408.

11. Ertek S, Cicero A (2013): Impact of physical activity on inflammation: effects on cardiovascular disease risk and other inflammatory conditions. Arch Med Sci 8: 794-804.

12. WHO: Definition and diagnosis of diabetes mellitus and intermediate hyperglycemia: Report of a WHO/IDF Consultation. NLM classification: WK 810. WHO, Geneva 2006.

13. American Diabetes Association (2013): Standards of medical care in diabetes - 2013. Diabetes Care 36: S11-S66.

14. McPherson RA, Pincus MR (eds.) (2007): Henry's Clinical Diagnosis and Management by Laboratory Methods. $21^{\text {st }}$ ed. Saunders Elsevier, Philadelphia: 1400-1409.

15. Schosinsky KH, Lehmann HP, Beeler MF (1974): Measurement of ceruloplasmin from its oxidase activity in serum by use of o-dianisidine dihydrochloride. Clin Chem 20: 1556-1563.

16. Haack M, Sanchez E, Mullington JM (2007): Elevated inflammatory markers in response to prolonged sleep restriction are associated with increased pain experience in healthy volunteers. Sleep 30: 1145-1152.

17. Arnardottir ES, Mackiewicz M, Gislason T, et al. (2009): Molecular signatures of obstructive sleep apnea in adults: a review and perspective. Sleep 32: 447-470.

18. Can M, Acikgoz S, Mungan G, et al. (2006): Serum cardiovascular risk factors in obstructive sleep apnea. Chest 129: 233-237.

19. Lui MM, Lam JC, Mak HK, et al. (2009): C-reactive protein is associated with obstructive sleep apnea independent of visceral obesity. Chest 135: 950-956.

20. Taheri S, Austin D, Lin L, et al. (2007): Correlates of serum C-reactive protein (CRP) - no association with sleep disordered breathing. Sleep 30: 991-996.

21. Engström G, Hedblad B, Stavenow L, et al. (2004): Fatality of future coronary events is related to inflammation-sensitive plasma proteins: a population-based prospective cohort study. Circulation 6: 27-31.

22. Turnlund JR (1998): Human whole-body copper metabolism. Am J Clin Nutr 67: 960S-964S.

23. Araya M, Olivares M, Pizarro F, et al. (2003): Copper exposure and potential biomarkers of copper metabolism. BioMetals 16: 199-204.

24. Cofta S, Wysocka E, Torliński L, Goździk J (2005): Copper and zinc concentrations in blood of patients with obstructive sleep apnea syndrome. Trace Elem Electr 1: 1-5.

25. Volna J, Kemlink D, Kalousova M, et al. (2011): Biochemical oxidative stress-related markers In patients with obstructive sleep apnea. Med Sci Monit 17: CR491-497.
26. Dash PK, Redell JB, Hergenroeder G, et al. (2010): Serum ceruloplasmin and copper are early biomarkers for traumatic brain injury-associated elevated intracranial pressure. J Neurosci Res 88: 1719-1726.

27. Baysal E, Taysi S, Aksoy N, et al. (2012): Serum paraoxonase, arylesterase activity and oxidative status in patients with obstructive sleep apnea syndrome (OSAS). Eur Rev Med Pharmacol Sci 16: 770-774.

28. Majewski W, Iskra M, Stanisic M, et al. (2007): The importance of ceruloplasmin oxidase activity in patients with chronic lower limb atherosclerotic ischemia. Int Angiol 26: 341-345.

29. Bieli P, Calabrese L (2002): Structure to function relationships in ceruloplasmin: a 'moonlighting' protein. Cell Mol Life Sci 59: 1413-1427.

30. Kedziora-Kornatowska K, Czuczejko J, Szewczyk-Golec K, et al. (2006): Effects of perindopril and hydrochlorothiazide on selected indices of oxidative stress in the blood of elderly patients with essential hypertension. Clin Exp Pharmacol Physiol 33: 751-756. 